\title{
Supuestos epistémicos en el análisis de la movilidad social
}

\author{
Epistemological assumptions on social mobility's analysis \\ Jésica Lorena Pla / jpla@sociales.uba.ar \\ Universidad de Buenos Aires, Consejo Nacional de Investigaciones Cientificas y \\ Técnicas (CONICET), Argentina
}

\begin{abstract}
The main objective of this article is to identify the hegemonic perspective on social mobility studies, the political assumptions of this, and present an alternative. This alternative would allow us to use the social mobility concept but regarding the study of the social class structuring processes. Particularly, we try to show how both alternatives have different explanations for social inequality on Latin American societies. A synthesis of the functionalist vision is presented, in association with the interpretative and political inferences that emerge from it. Then, an alternative proposal that allows us to consider social mobility in the field of class structuring processes, in which social policies have a key role by promoting different gradients of income distribution, with regressive or redistributed effects on class inequality. Class paths appear as the concept that joins these processes within a relational view of social classes.
\end{abstract}

Key words: mobility, social classes, political meanings, social inequality, social issue.

Resumen: El presente artículo tiene como objetivo general identificar la corriente que hegemonizó los estudios de movilidad social desde sus inicios, las implicancias políticas de la misma (en relación con la idea de igualdad), y a la par presentar una alternativa para no desechar la idea de movilidad social, pero insertarla en el estudio complejo, relacional y combinado de los procesos de estructuración de clase. De manera particular, buscamos dar cuenta cómo ambas propuestas tienen efectos diferenciales a la hora de explicar los procesos de igualdad-desigualdad en las sociedades latinoamericanas contemporáneas. Se presenta una síntesis de la postura estructural funcionalista sobre la movilidad social, poniéndola en relación con las inferencias (interpretativas y políticas), que se pueden hacer a partir de la misma y luego una propuesta alternativa para pensar la movilidad social en el ámbito de los procesos de estructuración de clases en los cuales las políticas sociales tienen un rol fundamental, al promover diferentes gradientes de distribución del ingreso, entre otros elementos, con efectos regresivos o redistribuidos sobre la desigualdad de clase. Las trayectorias de clase aparecen como el concepto que conjugarían dichos procesos, dentro de una mirada relacional de las clases sociales.

Palabras clave: movilidad, clases sociales, significaciones políticas, desigualdad social, cuestión social. 


\section{Introducción}

En América Latina, durante los últimos diez años, el estudio de los procesos de movilidad social ha tenido un nuevo protagonismo, ${ }^{1}$ luego de décadas en los cuales el tema estuvo ausente del debate académico en el campo de las ciencias sociales. En un marco de cambios políticos, económicos y sociales, la idea de movilidad social no sólo volvió a estar en boga en dicho ámbito, sino que atraviesa las discusiones políticas (y sobre políticas sociales), en tanto se le considera un valor central respecto a la integración y la cohesión social.

De este modo, suele aparecer una valoración positiva del concepto, generalmente asociada a una idea de igualdad, de una sociedad que garantiza oportunidades y recompensas a sus ciudadanos. Esta interpretación, naturalizada y puesta en escena como de "sentido común", lleva implícito un paradigma teórico y una interpretación política sobre la desigualdad social que debe ser explicitada, a la hora de utilizarla en el debate académico. Los estudios realizados hasta el momento han circunscrito sus análisis a la relación entre origen social (de clase, de posición) y el "destino" de una persona (de clase, de posición, generalmente ocupacional).

Sin embargo, sin negar la riqueza de dichos estudios, y los procesos que iluminan, sostenemos que es necesario poner este tipo de abordajes en relación con los procesos sociales de mayor envergadura que acucian a la región, y de ese modo comprender los procesos de movilidad social intergeneracional en el marco de procesos de estructuración de clases sociales (espacios sociales), en el cual se conjugan diferentes elementos, como veremos a continuación, que permiten dar cuenta de mejor manera de procesos sociales centrales, y ponerlos en relación con otros, tales como distribución del ingreso, consumo, pobreza, riqueza, desigualdad.

1 La compilación de Franco, León y Atria (2007b) es una exposición de los principales textos sobre estratificación y movilidad de este periodo, con trabajos sobre Brasil (Valle Silva), Argentina (Mora y Araujo, Kessler y Espinoza); León y Martínez por un lado, y Torche y Wormald por otro, para el caso de Chile; Cortés y Escobar Latapí para México; Pérez Saín y otros para Centro; y Gray Molina y otros para el caso de Bolivia. Otras producciones relevantes han sido las de Benavides (2002), Boado (2008), Cortés y Solís (2006), Costa Ribeiro (2007), Fachelli y López Roldán (2012), Núñez y Risco (2004), Solís (2004; 2011). En el caso de Argentina, también ha habido un renovado interés. Durante las dos décadas anteriores a la actual, sólo Jorrat $(1987,1997,2000,2005,2007,2011$ a) abordó estas temáticas. Más recientemente, muchos autores han tocado estos temas. Una buena síntesis de dicho proceso puede encontrarse en AAVV (2011) y en IIGG (2011). 
Para pensar esa crítica, es necesario dar cuenta no sólo de los procesos de movilidad social, sino del modo en el cual los mismos se insertan en la relación entre la estructura de clases, los contextos económicos, sociales y políticos y las dimensiones que permitirían dar cuenta de diferentes patrones de igualdad-desigualdad (Pla, 2012). La movilidad entonces no puede ser pensada como un indicador de igualdad, pues existe la posibilidad de que existan sociedades altamente móviles y altamente desiguales (Jorrat, 2005).

Por ese motivo sostenemos que la discusión sobre los procesos de movilidad social debe estar enmarcada en una perspectiva relacional de las clases sociales, en la cual las trayectorias de movilidad sean indicadores de procesos donde estructura y agencia se imbrican junto a los factores antes mencionados, dando lugar a espacios sociales en los que las posiciones objetivas se construyen con y a la par de posiciones subjetivas. Para hacer esta tarea es necesario especificar la perspectiva teórico epistemológico que subyace a la idea de movilidad social, pues por mucho que se ha escrito sobre ella, aparece generalmente invisibilizada, incluso desde ámbitos académicos.

El surgimiento y consolidación de los estudios de movilidad social tuvo lugar dentro de un campo epistemológico específico: el estructural funcionalismo. De manera sintética y para nada exhaustiva, ${ }^{2}$ el mismo sigue una línea de desarrollo que comienza en Saint Simon, pasa por Auguste Comte y Emile Durkheim, y llega a Talcott Parsons (Giddens, 1979). El argumento que subyace y da forma a dicho paradigma sostiene que las necesidades de un grupo social eclipsan las individuales. Esta concepción tiene su origen en el concepto de división del trabajo de Durkheim, el cual no deja espacio al conflicto o la lucha de clases (Feito Alonso, 1995: 45).

El proceso de estratificación social aparece entonces como un mecanismo que garantiza la "necesidad" del sistema social de que las posiciones más importantes de la sociedad la ocupen las personas "más" cualificadas y competentes, quienes a su vez recibirán como recompensa una mayor participación en la distribución de bienes sociales (escasos).

Esta concepción sobre la relación estructura e individuo entraña también un valor de incentivo al esfuerzo por el ascenso social. Si las necesidades del sistema social eclipsan las individuales, ¿cómo se determina quiénes son los mejores y más competentes para ocupar los puestos más relevantes? Acorde con esta concepción, el proceso se ejecuta por medio de un mecanismo de competencia, que, partiendo de la igualdad de oportunidades en el origen (igualdad jurídica, igualdad de oportunidades en términos de libertad liberal),

2 Sobre estos debates puede consultarse Laurin Frenette (1989), Cachón Rodríguez (1989), y en particular sobre la relación con la movilidad social Pla (2013). 
los individuos ponen en juego diversas motivaciones que determinan gradientes diferenciales de esfuerzos. A mayor esfuerzo, mayor capacidad en esa competencia por ocupar el lugar más valorado de la estructura social y, en consecuencia, mejores recompensas.

Esta visión, que reconoce sus postulados básicos en la obra de Parsons, eclipsó el pensamiento social durante las dos décadas posteriores a la segunda guerra, periodo que se ha denominado de "consenso ortodoxo" (Feito Alonso, 1995: 32). Es en los años setenta ${ }^{3}$ cuando se suma al debate la postura neoweberiana (Goldthorpe 1987, 1992) y un poco más tardíamente la neomarxista (Wright, 1997).

Decimos entonces que si la teoría de la movilidad social nace impregnada de los supuestos de la teoría estructural funcionalista, cabe especificar qué significan sus estudios, qué nos dicen sobre los procesos sociales que atraviesan a las sociedades latinoamericanas, en relación con dimensiones centrales como la desigualdad, la pobreza, la distribución del ingreso, etc. En esta línea, nos proponemos, en el presente artículo, hacer un somero repaso sobre el modo en que los estudios de movilidad social se insertan en una corriente hegemónica en las décadas de posguerra, y en un modo particular de comprender no sólo los procesos de estratificación, sino también el modo en que se utilizan en nuestro continente.

Para hacerlo revisaremos las concepciones que rodean el surgimiento de los estudios de movilidad social, el modo en que éstos se aplican en América Latina, pero también las implicancias teóricas que llevaban en sí estos estudios respecto al ya mencionado lugar que el continente ocupa a nivel mundial.

Nuestro objetivo es identificar la corriente que hegemoniza los estudios de movilidad social, las implicancias políticas de la misma (en relación con la idea de igualdad), y presentar una alternativa para no desechar la idea de movilidad social, pero insertarla en el estudio complejo, relacional y combinado

3 El VII Congreso Mundial de Sociología en Varna (Bulgaria) es un punto de inflexión en este consenso ortodoxo (Cachón Rodríguez, 1989: 181). Durante el mismo se presenta una serie de comunicaciones que se pueden dividir según sigan dentro del campo teórico del funcionalismo o se cuestionen la validez del mismo. De entre los primeros se destacan Jones, Sorensen y Rishöj. Entre los que se cuestionan los postulados del funcionalismo se destaca Daniel Bertaux. Por su parte, Goldthorpe sostiene que puede haber una "tercera" perspectiva que se representa en el grupo del Nuffield College de Oxford, la cual desarrollaremos más extensamente en el apartado siguiente. Paralelamente en la vieja Europa después del X Congreso del PCUS el marxismo occidental adquiere un nuevo vigor, en particular por el impacto del estructuralismo de Levi-Strauss y la relectura de algunos clásicos como Gramsci. Esto implica el análisis de temas fundamentales de la sociología desde la perspectiva del materialismo histórico: la economía, el Estado, la sociedad, el trabajo. 
de los procesos de estructuración de clase. De manera particular, buscamos dar cuenta de cómo ambas propuestas tienen efectos diferenciales a la hora de explicar los procesos de igualdad- desigualdad en las sociedades latinoamericanas contemporáneas.

\section{Repensar la movilidad social. Modernización y desarrollo. La visión relacional}

En el apartado anterior señalamos que durante las décadas de la posguerra la teoría funcionalista sobre la estratificación social tuvo su periodo de hegemonía. Es en esa misma época, y bajo la misma esfera de sentidos (epistémica), que el concepto de desarrollo (económico) deviene un concepto central para explicar los procesos nacionales y las relaciones internacionales entre países y regiones. Dicho concepto sirvió para dividir al mundo en dos áreas: los "desarrollados" (países centrales) y los "en vía de desarrollo" (países periféricos, en particular Latinoamérica).

¿Qué significaba, en términos políticos y de relaciones internacionales, esta división? Si existían unos países desarrollados, y otros "en vías de desarrollo", estos últimos debían seguir un camino que los identificara con los primeros (Quijano, 2000). Dicha división se constituye como una de las expresiones de la reconfiguración del poder capitalista mundial y lo hace con base en reconstruir un patrón eurocéntrico de conocimiento que desde el siglo XVIII se constituyó como uno de los instrumentos principales del patrón mundial de poder y dominación capitalista (Quijano, 2000). ${ }^{4}$

En Latinoamérica existieron dos vertientes que se enmarcaron en esta línea: la teoría de la modernización (Franco, León y Atria, 2007a) y las teorías dependentistas. ¿Cómo opera ese mecanismo de dominación? En el caso de la teoría de la modernización se basó en dividir a la humanidad en "áreas" y en otorgarle "la cultura" la condición de ser fuente y explicación de las diferencias entre los grupos humanos respecto del desarrollo.

De este modo, alcanzar el desarrollo supone seguir las pautas culturales de las áreas desarrolladas, diferencias que se naturalizaron al darlas por sentadas (Quijano, 2000). El núcleo central de esta teoría era explicar la crisis de transición desde el capitalismo agrario al capitalismo industrial que atravesaba la mayoría de los países de la región en el periodo de la posguerra (Franco, León y Atria, 2007a: 27).

4 Una revisión exhaustiva de este proceso se puede encontrar en Escobar (1999) y en Faletto (2009). 
Es este punto aparece la idea de movilidad social. La movilidad social sería, en dicha visión, el proceso que caracteriza a los países desarrollados, pues pone en evidencia mecanismos "libres" de competencia, en condiciones de igualdad jurídica, para alcanzar los diferentes puestos de un sistema social.

Es el estatus o el honor lo que está en la base de la estratificación social, los actores siguen los valores de la sociedad y con el objetivo de satisfacer las necesidades del sistema social: no es que los individuos busquen la riqueza, sino que ésta es una recompensa secundaria a quien más se esforzó por vivir según las necesidades de la sociedad y así aportó en un mayor grado a la integración del sistema social. La estratificación tiene entonces una función integradora y adaptativa, una función moral (Parsons, 1968).

Esta mirada se sustenta en la distinción que hace Durkheim entre conciencia individual y conciencia colectiva. Mientras que la primera refiere al ámbito privado de una persona, la segunda refiere a un aspecto más macro y es definida como formas de obrar, pensar, sentir que integran una sociedad y se transmiten de generación en generación: se trata de la orientación normativa de la acción en el estructural funcionalismo de Parsons (Feito Alonso, 1995).

Dos de los principales exponentes de los estudios de movilidad social de posguerra, Lipset y Bendix (1963), sostuvieron que las diferencias en la movilidad social entre los países se vinculan al ritmo de urbanización e industrialización de los mismos. Existe una pauta convergente de desarrollo que suponía que una vez que los países "entran" en un tipo avanzado de industrialización (desarrollo), tienden a hacerse cada vez más comparables en sus organizaciones institucionales y en sus sistemas sociales. Éstos se caracterizan por ser sistemas abiertos, particularmente "meritocráticos" (siendo el sistema educativo el mecanismo de selección de las personas para los puestos).

Fue Treiman quien sistematizó los supuestos de esta corriente ${ }^{5}$ en relación con los procesos de movilidad (y estratificación) social. Sintéticamente, dichos postulados pueden resumirse en los siguientes puntos básicos:

- Cuanto más industrializada (desarrollada) una sociedad, menor influencia directa de la posición -estatus- ocupacional del padre sobre la posición del hijo,

- pero también en la formación educativa, mayor la influencia de las cualificaciones educacionales, mayor la influencia del estatus ocupacional en la renta, menor la influencia directa de la educación en la renta, menor la correlación entre educación y renta.

- Cuanto más industrializada una sociedad, mayor la tasa de movilidad de intercambio, particularmente cuanto más alto el nivel educativo,

5 En su obra "Industrialization and social stratification", en Laumann (1970: 221). 
la distribución de los medios de comunicación, el nivel de urbanización y la movilidad geográfica.

Es decir, desarrollo y movilidad social aparecen relacionados, siendo ambos características de las sociedades modernas, a las cuales los países subdesarrollados debían aspirar, siguiendo el modelo y las pautas de valores culturales que subyacen en los mismos. Esas pautas culturales a imitar serían los sistemas de valores compartidos que motivan a los individuos a esforzarse (educarse) en pos de alcanzar los puestos más altos, socialmente más reconocidos, de la estructura social.

Esta perspectiva pone el foco en el individuo como responsable de su propio destino, ante un sistema "libre" (de libre mercado, sin restricciones políticas-institucionales), los individuos trazan caminos de movilidad social por medio de esfuerzos que los llevan a diferentes logros, desigualmente reconocidos, desigualmente recompensados.

Las sociedades modernas son entonces no sólo las sociedades desarrolladas económicamente, en las cuales a la postre prima la economía de mercado como vector de la sociedad, sino aquellas donde ese predominio de la economía de mercado, de la libertad en el sentido liberal, asume la forma de un sistema compartido de valores que regulan las relaciones entre los hombres y sirve como marco, explicación y justificación de las desigualdades sociales.

Los orígenes de los estudios de movilidad social en América Latina se dan a la par de estos postulados (Acevedo Rodríguez, 2009: 13). Entre las décadas de 1960 y 1970 se realizaron en la región análisis comparativos de estratificación y movilidad social, en particular en las ciudades de San Pablo, Buenos Aires, Montevideo, Santiago de Chile y Perú. ${ }^{6}$ Dichos estudios estuvieron centrados en analizar, como ya dijimos, las consecuencias del pasaje de una sociedad agraria a una industrial, suponiendo que la aparición de esta última daría paso a una sociedad moderna en la cual la frontera entre los estratos tendían a difuminarse, y el conjunto de la sociedad adquiría el carácter de un continuum (Faletto, 2009: 224).

Estos argumentos se sustentan, de manera implícita, en la teoría de la modernización, y asocian a esas ideas el distinguir como elementos importantes del análisis de la estratificación social la transformación estructural de las economías y los cambios concomitantes en la estructura social, tanto respecto

6 Costa Pinto $(1956 ; 1959)$ y Bresser Pereira (1964) son los principales representantes de Brasil. Germani (1963) en Argentina. En Uruguay los estudios destacados los realiza Solari, por su cuenta en 1956, y junto a Labbens en 1966. En Perú destaca Chaplin (1968). Para el caso de Chile encontramos a Raczynski (1971; 1974) y Hutchinson (1962), quien sintetiza un estudio comparativo de Santiago de Chile, Montevideo y Buenos Aires. 
al surgimiento, declinación o desaparición de lo que podrían llamarse "grupos funcionales", como a las relaciones de poder que se establecen entre los distintos grupos y clases (Faletto, 2009: 226).

De manera concreta, se señalaba que las sociedades producto de la modernización tendrían un importante predominio de clases medias, dado que las diferencias entre estratos serían tenues y continuas, y existiría movilidad social con base en un elemento principal, la educación (Franco, León y Atria, 2007a: 28). Asimismo, desde esta perspectiva, las clases medias fueron visualizadas y ubicadas como los agentes de cambio para la modernización.

Los estudios específicos sobre movilidad social estuvieron entonces bajo la órbita del pensamiento de la modernización o estructural funcionalista. La idea de movilidad social refiere a un proceso, de estratificación, en el cual individuos "libres" acceden al mercado, en búsqueda de diferentes "puestos", que se dividen de manera diferencial según las necesidades del sistema social, y que, en consecuencia, son desigualmente recompensados.

El sistema educativo (que actúa también bajo un supuesto de igualdad en el acceso), funciona como el mecanismo de selección para los diferentes puestos. Movilidad aparece entonces como igualdad. Uribe Mallarino (2005: 41-42) señala que en esta mirada la existencia de capas sociales no implica la lucha de clases que era central en la visión marxista. Por el contrario, son el talento y las habilidades naturales unidos al esfuerzo y la oportunidad, además de la posición heredada, los factores que explican la movilidad.

\section{Repensar la movilidad social}

Tal como señala Filgueira $(2001,2007)$, los primeros estudios sobre movilidad social en América Latina, antes mencionados, tuvieron la particularidad de registrar con precisión los efectos positivos del desarrollo económico y productivo sobre los patrones de movilidad social intergeneracional, y también fueron especialmente exitosos en reconocer los efectos de la inmigración a la ciudad, la caída de la ocupación en el sector primario, el creciente grado de "salarización" de la población económicamente activa, y la expansión del sistema educativo, procesos de particular interés en ese momento histórico.

Sin embargo, el paradigma desde el cual se hizo estuvo fuertemente sesgado por una visión liberal del orden social, la distribución del poder y el prestigio, y por una orientación centrada en observar los sistemas económicos y la influencia del contexto internacional sobre el desarrollo económico y social de los países. 
De este modo, sostenemos que un paradigma alternativo para estudiar los procesos de estratificación social debe superar las limitaciones del paradigma clásico, sesgado hacia los mecanismos de mercado y los condicionamientos externos, y recuperar la idea de "estructura de oportunidades", es decir, el modo en el cual se distribuyen las oportunidades para el acceso a posiciones sociales diferencialmente evaluadas. De manera particular, el modo en que se procesan los procesos macroestructurales al interior de cada país por medio de políticas (de welfare). ¿Por qué sostenemos esto?

La relación capital-trabajo, que estructura a las sociedades capitalistas, es una relación (desigual) mediada, desde sus orígenes, por la acción de políticas que moldean la cuestión social, la desigualdad inherente al sistema de clases, articulando aspectos re-distributivos o regresivos que modifican, positiva o negativamente, la distribución que se produce de forma directa por la vía del mercado.

Las políticas (sociales) son entonces diseñadas para incentivar o conservar la estructura de desigualdad que induce el mercado (Nolan et al., 2010), convirtiéndose así en una de las variables más relevantes para entender las diferentes estructuras de oportunidades. Las intervenciones estatales no son azarosas, sino que responden a una concepción, social y políticamente convalidada, tanto en la definición de los sujetos destinatarios como en la representación social de la vida cotidiana, el rango de variación y los contenidos de tales diferencias (de la desigualdad) (Danani, 2005: 21). Serán los gradientes en el elemento desmercantilizador / mercantilizador (Esping-Andersen, 1993) los que determinen impactos diferenciales en la estructura de clase.

Recuperar esta mirada no implica sólo incorporar "una variable" al análisis de la movilidad social (muchas veces hecho por medio de comparar "periodos históricos" que refieren a distintos contextos políticos, pero sin contextualizar los mismos), sino dar cuenta de que no existe una relación lineal entre movilidad e igualdad, que las políticas tienen efectos de estratificación.

Se trata de concebir a los procesos de movilidad social desde una mirada relacional de las clases sociales (en oposición a la "gradacional" centrada en el estatus, propia del paradigma estructural funcionalista). Abordar estos procesos desde dicha mirada significa que las diferentes clases conforman un sistema de dependencia y que su definición está dada por las relaciones sociales que se estructuran entre clases: no se trata de que una clase sea "menos" que la otra, sino que ocupan una posición social diferenciada y desigual en un sistema, por ejemplo, el mercado de trabajo. Todas las definiciones de clase social que se estructuran en torno a esta idea, entre las cuales pueden ubicarse las concepciones marxistas y weberianas, coinciden en que las estructuras sociales desiguales conforman, a su vez, estructuras de intereses (Feito Alonso, 1995: 31). 
Tanto la concepción marxista como la weberiana reconocen a la esfera económica como el espacio de constitución de las clases sociales, como una esfera determinante del orden social: para unos las clases son resultado de las relaciones de producción, para otros de las oportunidades de los sujetos de valorar en el mercado los recursos que poseen (Longhi, 2005: 106).

Dentro de estas teorías, el concepto de clase social permite ubicar a los individuos en un lugar determinado dentro de una estructura social determinada, y reconocer las relaciones y mecanismos de control, conflicto y lucha que se generan entre las distintas clases, la diferencialidad de posiciones y el conflicto en torno a ella. Desde dicha mirada la clase social da cuenta de un fenómeno temporal de la estratificación.

De manera particular, esta mirada nos permite concebir a la clase social como un espacio social caracterizado por el conjunto de posiciones — distintas y coexistentes - de los agentes. La clase social no se define entonces por una propiedad específica (como la posesión de los medios de producción) ni por una suma de propiedades en una relación de causa a efecto, sino por la estructura de las relaciones entre todas las propiedades pertinentes.

Esta clasificación se construye a partir de la identificación de las posiciones que los agentes ocupan en relación al capital económico, simbólico, cultural y social. Entre esas dimensiones se configuran procesos sociales de movilidad, que evidencian trayectorias, pero que a la par con otros componentes pueden asumir diferentes características. Es posible observar reproducción de clase, en términos intergeneracionales, pero espacios sociales con mayores capacidades de adquirir capitales económicos (en la lucha por la distribución del ingreso), es decir, con mayor capacidad de influencia en la distribución del ingreso, o a la inversa, según los componentes políticos del contexto sociohistórico.

En este sentido, la política social constituye una estrategia de intervención, desde el Estado, que interviene sobre las relaciones sociales, y tiene efectos de configuración de la estructura social. La incorporación de estas dimensiones permite analizar aspectos fundamentales de la estratificación social, en el sentido de que permiten delimitar los criterios de diferenciación, más allá del mercado, así como la mutación de los mismos en el proceso histórico.

Es decir, permite evaluar no sólo la movilidad individual sino también los procesos de cambio dentro del espacio social, los cuales aluden a procesos masivos que alteran el destino de ciertos grupos, clases o segmentos de la sociedad (Filgueira, 2007). 


\section{La movilidad social desde las trayectorias intergeneracionales de clase y el espacio social}

De manera general, el debate sobre la estratificación y la movilidad social ha sido abordado desde tres paradigmas, siendo el estructural funcionalista el que más hegemonía y difusión ha tenido. Ahora bien, como señala Cachón Rodríguez (1989: 528), la sociología funcionalista no satisface las condiciones del problema que aborda y, en consecuencia, no es la teoría científica general que pretende ser. Es por esta razón que deberá ser otra sociología la que asuma el papel de intérprete de los procesos de movilidad social.

Su principal objetivo deberá ser romper con los supuestos del funcionalismo, las implicancias políticas que éste lleva implícito, y reconstruir el campo hacia un nuevo paradigma, que por contraste con los cuatros aspectos nuevos supuestos: 1) La realidad no es transparente sino que la sociología hace que algo sea visible (interpretación); 2) la sociedad debe ser comprendida como un mercado segmentado, la sociología de la movilidad social ha de partir de alguna teoría de la segmentación del mercado de trabajo y no de una concepción homogénea del mismo; 3) los hechos sociales básicos del campo de la movilidad social afectan fundamentalmente a grupos sociales como tales, cuya condición varía en la estructura de posiciones sociales; 4) diversos mecanismos interrelacionados determinan la posición de los grupos sociales y de los individuos en su interior, así como sus posibilidades de modificar históricamente su posición (familia, escuela, mercado de trabajo, Estado y otros).

En conjunción con estos elementos aparece un quinto factor, que complementa esta visión crítica. Si las relaciones origen-destino habían sido pensadas desde la idea de movilidad, ahora deben ser pensadas desde la idea de la trayectoria de clase. Si la movilidad era parte integrante de la estratificación social (funcionalista), las trayectorias sociales son parte de las clases sociales (mirada relacional, como ya dijimos).

Y esto no quiere decir tan sólo que la sociología de las trayectorias sociales se ha de inscribir en una sociología de las clases sociales, sino que las trayectorias sociales son trayectorias de clases. La sociología de las trayectorias sociales deberá inscribirse en un marco teórico más amplio que considere los procesos que las enmarcan. Es decir, uno de los elementos que debe caracterizar a los estudios de las trayectorias de clase, de la movilidad social desde una perspectiva que impugne los supuestos del funcionalismo, debe incorporar una dimensión contextual, relacionar la situación de clase con la formación de estratos, articulando los diferentes órdenes de poder (económico, social y político) y analizando los efectos que, en momentos históricos concretos, tienen unos sobre otros. 
Además, para explicar los procesos de estructuración de clases en cada país es preciso dar cuenta de la inserción que cada contexto nacional tiene en el exterior, del "lugar" que ocupa en el sistema mundial y los modos como los países procesan, política mediante, esa posición.

Es decir, el estudio de las clases sociales, la movilidad social y los procesos de estratificación no puede explicarse sólo por las lógicas del mercado, sino que los mismos se introducen en un proceso de intervenciones políticas que implican aspectos, ya sea redistributivos o regresivos, orientadas a modificar la distribución que se produce por el mercado o diseñadas para incentivar o conservar la estructura de desigualdad que induce el mismo.

La hegemonía de la visión funcionalista sobre los estudios de la movilidad ha llevado a que los mismos sean desdeñados por los sociólogos que respondían a otras perspectivas. Sin embargo, ninguna de ellas logra conjugar los procesos sociales realmente existentes, las personas se mueven, transitan trayectorias diferenciales, constituyen espacios sociales que no son estáticos y en los cuales los capitales tienen no sólo diferente capital sino también diferente evaluación sobre los mismos.

Confluir con el análisis de movilidad desde una visión de clases (trayectoria) implica dar cuenta de un fenómeno que, a expensas de la reproducción social, existe: la sociedad de clases no es una sociedad de castas, es una sociedad "móvil" tanto en su estructura como en la cosmovisión del sentido común que atraviesa a los sujetos, producto de una construcción política propia.

La sociedad de clases es movilidad en tanto la misma idea ha sido construida como un vector de la igualdad simbólica. Estudiar estos procesos desde una visión de clase no implica aceptar esa justificación de la desigualdad basada en la igualdad de oportunidades, pero sí reconocerla y ponerla en cuestión en pos de comprender los mecanismos complejos que atraviesan a las sociedades de clases. Para ello, es necesario repensar los paradigmas teóricos desde los cuales se piensan los fenómenos, sobre todo las implicancias políticas que los mismos tienen.

Si la movilidad social es pensada desde la visión liberal estructural funcionalista, se observan individuos responsables de sus destinos, tanto de "éxitos" como de "fracasos", se responsabiliza a los últimos por su destino (justificado en la igualdad jurídica, la desigualdad es producto de desiguales esfuerzos y/o motivaciones personales), se reafirma el lugar de los primeros y se legitima la desigualdad de clases.

Incorporar el análisis de los procesos históricos, económicos y el modo en el cual la política interna de los países los conjuga en políticas de gobierno, implica desnaturalizar las desigualdades, ponerlas en cuestión y responsabilizar 
a colectivos mayores por las mismas. Implica además dar cuenta de las diferentes configuraciones territoriales que se dan en el continente, y de las especificidades del mismo en relación con los países centrales. Implica poner en juego aspectos internos e internos, y el modo en el cual se articulan en los procesos (macro micro), de estructuración de clases.

La mirada sobre la movilidad social entonces no puede estar escindida de una mirada sobre las clases sociales, sobre la desigualdad, sobre las formas específicas que asumen las mismas en cada periodo histórico (relativo a otros componentes, no sólo económicos, sino también simbólicos, culturales, sociales, etc.), sobre el modo en que las políticas operan sobre esas trayectorias, abriendo, cortando, cerrando o ciñendo caminos. La mirada sobre la movilidad social no puede asociar igualdad a movilidad, no sólo porque oculta la riqueza del fenómeno sino porque esconde la naturaleza desigual de las relaciones de clase en el sistema capitalista.

\section{Bibliografía}

AAVV (2011), Laboratorio Revista de Estudios sobre Cambio Estructural y Desigualdad Social, núm. 24, Mar del Plata: Ediciones Suárez.

Acevedo Rodríguez, Carlos (2009), "Germani y el estructural funcionalismo, evolucionismo y fe en la razón. Aspectos de la Involución Irracional”, en Revista Universum, núm. 24, vol. 1, México: Universidad de Talca.

Beigel, Fernanda (2006), "Vida, muerte y resurrección de las 'teorías de la dependencia", en Crítica y teoría en el pensamiento social latinoamericano, Buenos Aires: CLACSO.

Benavides, Martín (2002), "Cuando los extremos no se encuentran: un análisis de la movilidad social e igualdad de oportunidades en el Perú contemporáneo", en Boletín del Instituto Francés de Estudios Andinos, núm. 31, vol. 3, Lima, Perú.

Boado Martínez, Marcelo (2008), La movilidad social en el Uruguay contemporáneo, Montevideo: Instituto Universitario de Pesquisas do Rio de Janeiro (IUPERJ), Universidad Complutense de Madrid (UCM), Universidad de la República (Udelar), Comisión Sectorial de Investigación Científica (CSIC).

Borón, Atilio (2008), “Teoría(s) de la dependencia”, en Realidad Económica, núm. 238, vol. 16, Buenos Aires, Argentina.

Bresser Pereira, L. (1964), “The rise of middle class in Brazil", en Horowitz, L. [ed.], Revolution in Brazil: Politics and society in a developing nation, Nueva York: Dutton \& Co. Inc.

Cachón Rodríguez, Lorenzo (1989), ¿Movilidad social o trayectorias de clase?, Madrid: Siglo XXI Editores.

Chaplin, D. (1968), "Peruvian social mobility: revolutionary and developmental”, en Journal of Inter-American Studies, vol. 10, núm. 4, Miami, EUA.

Cortés, Fernando y Patricio Solís (2006), "Notas sobre la generación de información para los estudios de movilidad”, en Estudios Sociológicos, vol. XXIV, núm. 71, México.

Costa Pinto, E. (1956), "Social stratification in Brazil: a general survey of some recent changes", en Third World Congress of Sociology, Amsterdam. 
Convergencia Revista de Ciencias Sociales, núm. 71, 2016, Universidad Autónoma del Estado de México

Costa Pinto, E. (1959), "Estratificação social e desenvolvimento econômico", en Boletim do Centro Latino-Americano de Pesquisas em Ciências Sociais, vol. 2, núm. 3, Río de Janeiro.

Costa Ribeiro, Carlos Antonio (2007), “Class, race, and social mobility in Brazil”, en Dados, vol. 3, Río de Janeiro.

Danani, Claudia (2005), "La construcción sociopolítica de la relación asalariada: obras sociales y sindicatos en la Argentina, 1960-2000". Tesis de Doctorado en Ciencias Sociales, Facultad de Ciencias Sociales, Universidad de Buenos Aires. Inédito. Consultado en Biblioteca Norberto Rodríguez Bustamante.

Escobar, Arturo (1999), El final del salvaje. Naturaleza, cultura y politica en la antropología contemporánea, Bogotá: CEREC.

Esping Andersen, Gosta (1993), Los tres mundos del Estado del Bienestar, Valencia, España: Edicions Alfons el Magnánim.

Fachelli, Sandra y Pedro López Roldán (2012), “Two models of social stratification: from a classification scheme to a typology", Ponencia presentada en The Second ISA Forum of Sociology "Social justice and democratization", Buenos Aires, Argentina.

Faletto, Enzo (2009), Dimensiones políticas, sociales y culturales del desarrollo, Bogotá: Siglo del Hombre Editores-CLACSO.

Feito Alonso, Rafael (1995), Estructura social contemporánea. Las clases sociales en los países industrializados, Madrid: Siglo XXI Editores.

Fernández, Florestan (1973), "Problemas de conceptualización de las clases sociales en América Latina”, en Fernández et al., Las clases sociales en América Latina, México: Siglo XXI Editores.

Filgueira, Carlos (2001), "Estructura de oportunidades y vulnerabilidad social aproximaciones conceptuales recientes", documento preparado para el Seminario internacional "Las diferentes expresiones de la vulnerabilidad social en América Latina y el Caribe", Santiago de Chile.

Filgueira, Carlos (2007), “Actualidad de las Viejas temáticas: clase, estratificación y movilidad social en América Latina”, en Franco, Rolando, Arturo León y Raúl Atria [coords.], Estratificación y movilidad social en América Latina. Transformaciones estructurales de un cuarto de siglo, Santiago: LOM-CEPAL-GTZ.

Filgueira, Carlos y Andrés Peri (2004), América Latina: los rostros de la pobreza y sus causas determinantes, núm. 54, Santiago de Chile: CEPAL, Serie Políticas sociales.

Franco, Rolando, Arturo León y Raúl Atria (2007a), "Estratificación y movilidad social en América Latina. Una agenda de trabajo”, en Franco, Rolando, Arturo León y Raúl Atria [coords.], Estratificación y movilidad social en América Latina. Transformaciones estructurales de un cuarto de siglo, Santiago: LOM-CEPAL-GTZ.

Franco, Rolando, Arturo León y Raúl Atria [coords.] (2007b), Estratificación y movilidad social en América Latina. Transformaciones estructurales de un cuarto de siglo, Santiago: LOM-CEPAL-GTZ.

Germani, Gino (1963), "La movilidad social en Argentina”, en Lipset, S. y R. Bendix, Movilidad social en la sociedad industrial, Buenos Aires: Editorial Universitaria de Buenos Aires.

Giddens, Anthony (1979), La estructura de las clases en las sociedades avanzadas, Madrid: Alianza.

Goldthorpe, John (1987), Social mobility and class structure in modern Britain, Oxford: Clarendon Press. 
Goldthorpe, John (1992), "Sobre la clase de servicio, su formación y su futuro", en Revista Zona Abierta, núm. 59-60, Madrid.

Graciarena, Jorge (1973), “Comentario [a la propuesta de Fernández]”, en Fernández, Florestan et al., Las clases sociales en América Latina, México: Siglo XXI Editores.

Hutchinson, B. (1962), "Social Mobility rates in Buenos Aires, Montevideo and Sao Paulo: a preliminary comparison”, en Revista América Latina, año 5, Sao Paulo, Brasil.

IIGG (2011), Movilidad y cambio social en América Latina, Buenos Aires: IIGG [publicado en CD ROM].

Jorrat, Jorge Raúl (1987), "Exploraciones sobre movilidad ocupacional intergeneracional masculina en el Gran Buenos Aires”, en Desarrollo Económico, núm. 27, Buenos Aires, Argentina.

Jorrat, Jorge Raúl (1997), “En la huella de los padres: Movilidad ocupacional en el Buenos Aires de 1980”, en Desarrollo Económico, núm. 37, Buenos Aires, Argentina.

Jorrat, Jorge Raúl (2000), Estratificación social y movilidad: un estudio del área metropolitana de Buenos Aires, Tucumán: Universidad Nacional de Tucumán.

Jorrat, Jorge Raúl (2005), "Aspectos descriptivos de la movilidad intergeneracional de clase en Argentina: 2003-2004”, en Revista de Estudios sobre Cambio Social, año VI, núm. 17-18, Argentina: Instituto de Investigaciones Gino Germani, Facultad de Ciencias Sociales, Universidad de Buenos Aires.

Jorrat, Jorge Raúl (2007), "Movilidad intergeneracional de clase en Argentina 2002-2005”, ponencia presentada en el XXVI Congreso de ALAS, Guadalajara, México, 13-18 agosto.

Jorrat, Jorge Raúl (2008), “Exploraciones sobre movilidad de clases en Argentina: 20032004”, en Documentos de Trabajo, núm. 52, Buenos Aires: Instituto de Investigaciones Gino Germani, Facultad de Ciencias Sociales, Universidad de Buenos Aires.

Jorrat, Jorge Raúl (2010), "Los estudios de movilidad social de Germani. Aspectos descriptivos para el Gran Buenos Aires”, en Mera, Carolina y Julián Rebón [coords.], Gino Germani. La sociedad en cuestión. Antología comentada, Buenos Aires: Consejo Latinoamericano de Ciencias Sociales.

Jorrat, Jorge Raúl (2011a), "Diferencias de acceso a la educación en Argentina: 2003-2007”, en Lavboratorio Revista de Estudios sobre Cambio Estructural y Desigualdad Social, núm. 24, Mar del Plata: Ediciones Suárez.

Jorrat, Jorge Raúl (2011b), "Clase, identidad de clase y percepción de las sociedades desde elitistas a igualitarias: un estudio comparativo internacional", en CD ROM IIGG (2011), Movilidad y cambio social en América Latina, Buenos Aires: IIGG.

Laumann, E. O. [comp.] (1970), Social stratification: research and theory for the 1970's, Indianapolis: Bobbs Merrill, citado por Cachón Rodríguez, Lorenzo (1989), ¿Movilidad social o trayectorias de clase?, Madrid: Siglo XXI Editores.

Laurin Frenette, Nicolle (1989), Las teorias funcionalistas de las clases sociales: Sociología e ideología burguesas, España: Siglo XXI Editores.

Lipset, S. y R. Bendix (1963), Movilidad social en la sociedad industrial, Buenos Aires: Editorial Universitaria de Buenos Aires.

Longhi, Augusto (2005), "La teorización de las clases sociales", en Revista de Ciencias Sociales, año XVIII, núm. 22, Montevideo, Uruguay: Departamento de Sociología.

Marini, Ruy Mauro (1991), "Dialéctica de la dependencia”, en Dialéctica de la dependencia, México: Era. 
Núñez, Javier y Cristina Risco (2004) “Movilidad intergeneracional del ingreso en un país en desarrollo: el caso de Chile”, Documento de trabajo núm. 210, Chile: Departamento de Economía, Universidad de Chile.

Parsons, Talcott (1968), Hacia una teoría social de la acción, Buenos Aires: Kapeluz.

Pla, Jésica (2011), "Aproximaciones a los procesos de estratificación social en la Argentina Neoliberal y la Post Convertibilidad. Región Metropolitana Buenos Aires. 19952007/8", presentación en el Seminario Internacional "Movilidad y cambio social en América Latina”, 4-5 de noviembre de 2011 en la ciudad de Mar del Plata.

Pla, Jésica (2013), "Reflexiones sobre el uso del concepto de clase para el estudio de la movilidad social”, en Chávez Molina, Eduardo [comp.], Aportes a los estudios sobre desigualdad y movilidad social en el mundo contemporáneo, Buenos Aires, Argentina: Imago Mundi.

Quijano, Aníbal (2000), "El fantasma del desarrollo en América Latina”, en Revista Venezolana de Economía y Ciencias Sociales, vol. 6, núm. 2, mayo-agosto, Caracas, Venezuela.

Raczynski, D. (1971), Posición socioeconómica y consistencia de estatus de las ocupaciones, Santiago de Chile.

Raczynski, D. (1974), "La estratificación ocupacional en Chile”, en Los actores de la realidad chilena, Santiago de Chile: Editorial del Pacífico.

Solís, Patricio (2004), "Cambio estructural y movilidad ocupacional en Monterrey, México", ponencia presentada en el Primer Congreso de la Población de América Latina, ALAP, celebrado en Caxambú-MG- Brasil, 18-20 de septiembre de 2004.

Solís, Patricio (2011), "Desigualdad y Movilidad Social en la ciudad de México", en Estudios Sociológicos, vol. XXIX, núm. 85, México.

Torres Novoa, Carlos (1979), “Teoría de la dependencia: Nota crítica sobre su metodología histórico estructural”, en Nueva Sociedad, núm. 42, mayo-junio, Buenos Aires, Argentina.

Uribe Mallarino, Consuelo (2005), “Ascensos y descensos en la reproducción social”, en Universitas Humanistíca, año XXXI, núm. 59, Bogotá, Colombia.

Wright, Erik Olin (1997), Class Counts: Comparative Studies in Class Analysis, Cambridge: Cambridge University Press.

\section{Recursos electrónicos}

Nolan, Brian et al. (2010), “The role of social institutions in intergenerational mobility”, en Demosoc Working Paper, Paper Number 36, Barcelona. Disponible en: http://dcpis. upf.edu/ gosta-esping-andersen/materials/social_institutions.pdf. [1 de noviembre de 2014].

Pla, Jésica (2012), "Percepciones sobre la movilidad social y la planificación de trayectorias: Un enfoque biográfico sobre las incertidumbres al interior del hogar. Región Metropolitana Buenos Aires (Insights about social mobility and path planning: A biographical approach about the uncertainties within the household. Buenos Aires Metropolitan Region. 2011)", presentación en The Second ISA Forum of Sociology "Social justice and democratization", Buenos Aires, Argentina, 1-4 de agosto de 2012. Disponible en: http://www.riskanduncertainty.net/node/54 [12 de abril de 2013]. 
Jésica Lorena Pla. Doctora en Ciencias Sociales, Universidad de Buenos Aires. CONICET (Consejo Nacional de Investigaciones Científicas y Técnicas) con sede de trabajo en el IIGG (Instituto de Investigaciones Gino Germani, Facultad de Ciencias Sociales, Universidad de Buenos Aires, Argentina). Líneas de investigación: movilidad social, clases sociales, cuestión social, y la relación entre los procesos socio políticos y el proceso de estructuración de clases. Publicaciones recientes: "Consumo y trayectorias de clase. Distinción y competencia en el abordaje de los procesos de estratificación", en Revista Question, vol. 1, núm. 43, Argentina: Universidad Nacional de La Plata, Instituto de Investigaciones en Comunicación (IICOM) (2014); "Modernidad, desigualdad social e incertidumbre: apuntes para pensar los procesos de estratificación social desde una perspectiva dinámica", en Trabajo y Sociedad. Indagaciones sobre el trabajo, la cultura y las prácticas politicas en sociedades segmentadas, núm. 20, vol. XVIII (2013); "Cambio o continuidad: Una caracterización dinámica de las trayectorias inter-generacionales de clase. Región Metropolitana Buenos Aires. 1995-2007”, en Revista GPT (Gestión de las personas y la Tecnología), vol. 6, núm. 18, agosto, Chile: Universidad de Santiago de Chile (2013).

Recepción: 27 de noviembre de 2014.

Aprobación: 14 de enero de 2016. 
